\title{
Plural quantifiers: a modal interpretation
}

\author{
Rafal Urbaniak
}

Received: 17 August 2011 / Accepted: 23 September 2013 / Published online: 17 October 2013

C The Author(s) 2013. This article is published with open access at Springerlink.com

\begin{abstract}
One of the standard views on plural quantification is that its use commits one to the existence of abstract objects-sets. On this view claims like 'some logicians admire only each other' involve ineliminable quantification over subsets of a salient domain. The main motivation for this view is that plural quantification has to be given some sort of semantics, and among the two main candidates-substitutional and set-theoretic-only the latter can provide the language of plurals with the desired expressive power (given that the nominalist seems committed to the assumption that there can be at most countably many names). To counter this approach I develop a modal-substitutional semantics of plural quantification (on which plural variables, roughly speaking, range over ways names could be) and argue for its nominalistic acceptability.
\end{abstract}

Keywords Plural quantification - Nominalism · Higher-order logic · Boolos · Quantifiers · Tokens · Modality

\section{Introduction}

Examples of plural quantification are abundant in many natural languages (for instance, they occur in non-first-orderizable sentences like "Some logicians admire only each other"). Even though some people (like Boolos 1998a) intuitively take this sort of quantification to be nominalistically innocent, some (e.g. Hazen 1993) take a differ-

\footnotetext{
R. Urbaniak $(\varangle)$

Department of Philosophy, Gdansk University, Gdansk, Poland

e-mail: rfl.urbaniak@gmail.com

R. Urbaniak

Centre for Logic and Philosophy, Ghent University, Ghent, Belgium
} 
ent stance. One way to move the discussion forward is to provide a more elaborate philosophical and logical story explaining how plural quantification works on the nominalist's view and assess the nominalistic viability of this approach.

The goal of this paper is to tackle this issue by providing a modal semantics for plural quantification and arguing for its nominalistic acceptability. I start with a few brief remarks about the notion of ontological commitment, especially with reference to ontological commitment of logical systems (Sect. 2). Then, in Sects. 3 and 4, I introduce the language of the variant of the logic of plurals that I will be concerned with, quantified naming logic (QNL) and two standard semantical approaches to it. I discuss what I take to be the main argument to the effect that plural quantification bears commitment to the existence of sets in Sect. 5. It relies on the assumption that there are two candidates for adequate semantics for plural quantifiers: substitutional and set-theoretic (and since the former either doesn't provide the language with sufficient expressivity or doesn't satisfy certain prima facie compelling nominalistic restrictions, we are left with the latter and are thus committed to sets when we use plural quantifiers). First, I discuss these two candidates in Sect. 4. In Sect. 6, after describing this argument in Sect. 5, I counter the argument by developing a modal relational semantics for plural quantification which I find both theoretically and nominalistically viable. Sect. 7 is where I bring up some philosophical loose ends, defending the nominalistic acceptability of the whole enterprize.

\section{Logic and ontological commitment}

Before we turn to the question whether we are ontologically committed to sets when we use a certain type of quantification, a few words of explanation regarding the notion of ontological commitment are due. I take (interpreted) theories to be the primary bearers of ontological commitment. People become ontologically committed when they accept ontologically committed theories.

Quine's way of reading off the ontological commitment of a theory, in the stronger interpretation, consists of the conjunction of two claims: ${ }^{1}$

(i) Each such theory should be first translated into a regimented first-order extensional language.

(ii) The initially considered theory is committed to the existence of those things that have to belong to the range of first-order quantifiers of the regimented version thus obtained for that rendering of the theory to be true.

I tend to disagree with Quine [I mostly agree with the criticism of this sort of approach developed by Chihara (1973, 1990)]. Even putting (i) aside, I do not think there is a criterion of ontological commitment that both analyzes our intuitions about ontological commitment correctly and is epistemically more tractable than the notion of ontological commitment itself. It doesn't seem that our understanding of the relation ' $x$ has to be in the range of the first-order version of a theory $T$ for $T$ to be true' is any clearer that our notion of ontological commitment itself.

\footnotetext{
1 The weaker reading requires only that the language be first-order, without requiring that it be the extensional language familiar from first-order classical logic.
} 
One of the worries is, for instance, that many theories formulated in natural language, even if they have a first-order version, ${ }^{2}$ don't have a unique first-order version, and those non-unique versions tend to differ on what has to lie in their range of quantification in order for them to be true. ${ }^{3}$ True, we cannot always directly read off our ontological commitment from the way we ordinarily talk (if I do something for the sake of convenience, am I really committed to an object which is the sake of convenience?). But when we proceed with a first-orderization of our theories, our regimentation is often guided by some prior metaphysical conceptions that we are inclined to end up with anyway. Depending on what restrictions we put on our procedure we end up with different ontologies produced by the commitment of our first-order quantifiers. Agreement on theorems (modulo translation) is not enough to ensure the uniqueness of the underlying ontology. 4

However, I feel obliged to say a few words about ontological commitment. In particular, when does a logic commit one to sets? I adopt the following rough-andready criterion: a logic is committed to sets only if the adequate semantics for this logic requires some variables to range over sets (or that certain constants name sets) for theorems of this logic to be valid according to that semantics. If an (at least) equally successful account of theoremhood of this logic can be given which does not require the existence of sets, the logic (with this new semantics) does not commit one to sets. In other words, a logic carries commitment to sets if any philosophically viable semantics (which, at minimum, results in soundness, and ideally in completeness) requires that certain type of variables range over sets (or that certain constants refer to sets). ${ }^{5}$

\section{The language of QNL}

Quantified naming logic (QNL) will be the consequence operation that the language of QNL yields when given set-theoretic semantics (details will follow). ${ }^{6}$

\footnotetext{
2 The notion of being a first-order version is also not very clear, but let's not worry about that and just assume that what matters is agreement on theorems modulo some fixed translation.

3 A deeper investigation of these and related issues lies beyond the scope of my present considerations, though.

4 This point shines also through some of Peter Smith's remarks (2009) about (Parsons 2008).

5 I consciously ignore complications related to the distinction between preserving theoremhood and preserving derivability; these are not relevant in the current context.

6 Sometimes, I will use 'QNL' and 'the logic of plurals' interchangeably, trusting this will not cause any confusion, even though QNL differs slightly from more standard version of the logic of plurals. QNL can be also thought of as a part of Ontology — a logical system developed by Leśniewski (1930, 1931a,b), who developed a logical system called Ontology in early twentieth century, as a part of his attempt to develop a system of foundations of mathematics alternative to that of Principia Mathematica. The Leśniewskian twist to the debate is also worth bringing up because by the time the logic of plurals came to attention, a debate about the ontological commitment of plural quantification had already taken place, dressed up as a debate about interpreting Leśniewskian quantifiers. Nominalist as he was, part of Leśniewski's efforts was to construct his systems in a way that would render them nominalistically acceptable (that is, using them should not commit one to the existence of abstract objects). Hence, for instance, he went to great lengths constructing his axiomatized inscriptional syntax to ensure it does not bear commitment to expression types. Alas, he did not elaborate on the semantic aspect of the language of Ontology and treated quantification in Ontology as primitive. As the notion of formal semantics firmly established its position in the minds of mid-
} 
In QNL, name variables are the only admissible kind of variables and the only quantifiers are those that bind name variables. Well-formed formulas are constructed from name variables $a_{1}, a_{2}, a_{3}, \ldots \in \operatorname{Var}$, Boolean connectives $\neg, \wedge$, a sentential functor of two name arguments $\varepsilon$ (read as 'is one of' or simply 'is'), and the existential quantifier $\exists$ according to the following rules:

(i) If $\alpha_{1}, \alpha_{2} \in \operatorname{Var}$, then $\alpha_{1} \varepsilon \alpha_{2}$ is a well-formed formula.

(ii) If $\phi_{1}, \phi_{2}$ are wff's and $\alpha \in \operatorname{Var}$, then $\neg\left(\phi_{1}\right),\left(\phi_{1}\right) \wedge\left(\phi_{2}\right)$ and $\exists \alpha\left(\phi_{1}\right)$ are wff's. (iii) Nothing else is a wff.

Intuitively, name variables behave like place-holders for countable name phrases (henceforth called names), no matter whether those are empty, singular or refer to multiple objects. Under an interpretation ' $a \varepsilon b$ ' is true iff $a$ "names" exactly one object and this object is also "named" by $b$ ( $b$ may "name" other objects as well, but it does not have to). Some examples of QNL renderings of natural language sentences are: ${ }^{7}$

\begin{tabular}{ll}
\hline Socrates is a philosopher & Socrates $\varepsilon$ philosophers \\
All cats are animals & $\forall a(a \varepsilon$ cats $\rightarrow a \varepsilon$ animals $)$ \\
Some logicians admire & $\exists a[\forall b(b \varepsilon a \rightarrow b \varepsilon$ logicians $) \wedge$ \\
only each other & $\wedge \forall c \forall d(c \varepsilon a \wedge c$ admires $\wedge \wedge$ \\
& $\wedge d \varepsilon d \rightarrow \neg d \varepsilon c \wedge d \varepsilon a)]$ \\
\hline
\end{tabular}

\section{Semantics: set-theoretic and substitutional}

The main two alternative semantics for plural quantification are set-theoretic or substitutional. One of the main arguments for the claim that plural quantification commits one to sets relies on the claim that the substitutional interpretation is either theoretically or nominalistically unsatisfactory and that QNL with set-theoretic semantics carries commitment to sets. Let's review these semantics briefly.

A set-theoretic $Q N L$-model is a structure $\left\langle D, I_{\text {set }}\right\rangle$ where $D$ is a non-empty domain of objects and $I_{\text {set }}$ maps $V$ ar into the powerset of $D$. A substitutional QNL-model is a structure $\left\langle N, I_{s u b}, V a l\right\rangle$ where $N \neq \emptyset$ is a set of name substituents, $I_{\text {sub }}$ maps $V a r$ into $N$, and Val maps the set of pairs $\{\langle x, y\rangle \mid x, y \in N\}$ into $\{1,0\}$.

Under the substitutional interpretation the truth of substitution instances of atomic formulas is taken to be primitive and $\mathrm{Val}$ is a function that assigns truth values to such instances. Pure substitutional interpretation explicitly refuses to provide further analysis of truth-conditions of substitution instances of atomic formulas, espe-

\footnotetext{
Footnote 6 continued

twentieth century logicians, a question arose as to how we are to make sense of Leśniewski's quantification semantically so that it is clear that this quantification does not bear commitment to abstract objects (see for instance Lejewski 1954; Prior 1965; Küng and Canty 1970; Sagal 1973; Küng 1974; Kielkopf 1977; Küng 1977; Simons 1985; Rickey 1985; Simons 1995).

7 Notice that since terms don't have to be singular (nor do second arguments of 'admires'), it is better to use $d \varepsilon d$ in the antecedent of the translation of the last sentence, to make sure we say that any individual a logician from a given group admires belongs to that group. Presumably a member of this group admires the group itself as well, but we don't want to say that that group is a logician.
} 
cially in terms of the reference of their constituents. As for satisfaction, I skip the clauses for Boolean connectives and describe how it works for atomic and quantified formulas:

$$
\begin{aligned}
\left\langle D, I_{\text {set }}\right\rangle \models_{\text {set }} \alpha \varepsilon \beta \text { iff }\left|I_{\text {set }}(\alpha)\right|=1 \text {, and } I_{\text {set }}(\alpha) \subseteq I_{\text {set }}(\beta) . \\
\left\langle D, I_{\text {set }}\right\rangle \models_{\text {set }} \exists \alpha \phi \text { iff }\left\langle D, I_{\text {set }}^{\alpha}\right\rangle \models_{\text {set }} \phi, \\
\text { for some } I_{\text {set }}^{\alpha} \text { which differs from } I_{\text {set }} \text { at most at } \alpha . \\
\left\langle N, I_{\text {sub }}, \operatorname{Val}\right\rangle \models_{\text {sub }} \alpha \varepsilon \beta \text { iff } \operatorname{Val}\left(I_{\text {sub }}(\alpha), I_{\text {sub }}(\beta)\right)=1 . \\
\left\langle N, I_{\text {sub }}, \operatorname{Val}\right\rangle \models_{\text {sub }} \exists \alpha \phi \text { iff }\left\langle N, I_{\text {sub }}^{\alpha}, \operatorname{Val}\right\rangle \models_{\text {sub }} \phi, \\
\text { for some } I_{\text {sub }}^{\alpha} \text { which differs from } I_{\text {sub }} \text { at most at } \alpha .
\end{aligned}
$$

That is, set-theoretic semantics takes name variables to range over subsets of the domain, whereas the substitutional semantics takes an existentially quantified statement to be satisfied if one of its instances (with respect to the variable involved) is satisfied. $^{8,9}$

\section{The received view}

What I call the received view (motivated by certain remarks by Quine, Gödel or Skolem) is the view that any logic that uses higher-order quantification comparable to at least monadic second-order logic is committed to the existence of sets. One of the reasons why one might think that plural quantification bears commitment to abstract objects is the belief that plural quantifiers have to range over sets, presumably because another alternative, the substitutional reading of plural quantifiers is not satisfactory. One could imagine someone arguing as follows.

\footnotetext{
${ }^{8}$ It may seem that additional requirements should be put on $\mathrm{Val}$ so that certain formulas come out valid. For instance $\forall a, b, c(a \varepsilon b \wedge b \varepsilon c \rightarrow a \varepsilon c)$ is set-theoretically valid, but not substitutionally valid. (Take the interpretation where $N=\{1,2,3\}, \operatorname{Val}(1,2)=\operatorname{Val}(2,3)=1$ but $\operatorname{Val}(1,3)=0$, and $I(a)=1, I(b)=$ $2, I(c)=3$.) Actually, the point of the substitutional interpretation was to allow for disagreements of this sort (Dunn and Belnap 1968). For our present concerns, these issues aren't important.

${ }^{9}$ It is worth remarking that providing QNL with a set-theoretic semantics (arguably) deprives them of the expressive power that it has been argued the natural language plural quantification has. A classical argument is this. The following is intuitively true:
}

(4.1) There are some objects such that all and only those objects that are sets and not elements of themselves are among them.

The following, however, cannot be the case (the sentence states the existence of Russell's set):

(4.2) There is a set of objects such that all and only those objects that are sets and not elements of themselves are elements of it.

(4.1) seems to state the existence of a certain plurality (the plurality of those objects that are sets and not elements of themselves). So, prima facie, there are pluralities which are not sets. Hence, quantification over pluralities cannot be fully captured by interpretation which reads it as quantification over sets (or subsets of a domain).

For now, without any attempt to explain the use of plurals which does not seem to be captured by the set-theoretic semantics I will just remark that the expressivity provided by the set-theoretic semantics is complex enough to raise problems that will be my main concern. 
[SA1] Whenever we present a logical system (involving quantification) we have to provide it with a formal semantics which we think captures the meaning of these quantifiers adequately.

[SA2] If we use a certain logical system and believe that a certain semantics is an adequate semantics of this language, we are committed to whatever lies in the range(s) of quantification of some type of variables of the object language according to this semantics.

[SA3] The logic of plurals can have two kinds of semantics: a set-theoretic semantics (discussed before) or a substitutional semantics. ${ }^{10}$

[SA4] QNL with nominalistically acceptable substitutional semantics is not theoretically satisfactory.

[SA5] Therefore it has to be given a set-theoretic semantics.

[SA6] But on the set-theoretic reading of plural quantifiers, they range over subsets of a domain.

[SA7] Subsets of a domain are abstract objects and hence whenever we use the logic of plurals we are committed to abstract objects.

$\overline{10}$ For instance Kearns (1969, pp. 165-166) says:

I feel that one can distinguish two fundamentally different ways of regarding variables-I will call these two views of variables. The first view I call the Russell-Quine view; the second is the FregeLeśniewski view (these will be abbreviated as R-Q and F-L, respectively). These two are not the only possible views, but I feel that they are the two basic views; other views will be variants of one or the other, or perhaps combinations of the two...The R-Q view of variables could be called the pronoun view of variables. Professor Quine has compared the use of variables with many uses of pronouns in English; however, he views pronouns as more fundamental than nouns... On the R-Q view, quantifiers are used to talk about all entities or some entity...On the F-L view, a variable is seen as a replacement for an expression. Variables do not have ranges of values, where each value is an entity of some kind.

I am not convinced that Kearns is exactly right crediting the substitutional reading to Leśniewski; it seems that his interpretation relies on a mistranslation. Leśniewski in the passage Kearns refers to explains how he used quantifiers when he "did not yet know how to operate with quantifiers". It is therefore not sure whether this is how he understood his formal quantifiers once he had them. Also, he wrote "przy pewnym znaczeniu wyrazu", which translates as "for some meaning of the expression" or "for some meaning of the word" (Leśniewski 1927, p, 203). Kearns translates the phrase as "for some significant words" (which would be "dla pewnego wyrazu posiadającego znaczenie" or "dla pewnego znaczącego wyrazu" in Polish) and takes it to be Leśniewski's explanation of his quantifiers. But let's put these qualms aside. 
Let us dub arguments that go along these lines semantic arguments for the claim that higher order quantification commits one to abstract objects. ${ }^{11}$ For the sake of argument I will grant [SA1-2]. I will, however, take an issue with [SA4], and by dealing with it, also with [SA3].

Why exactly should we accept [SA4]? The classic paper which discusses problems with the nominalistic acceptability of the substitutional interpretation of higher-order quantifiers is (Küng and Canty 1970). ${ }^{12}$ The authors go through a certain variety of approaches to the problem and there are some bells and whistles that we won't look into. The main gist, however, is that no matter whether we think of names we can substitute as existing inscriptions, or whether we think of them as possible terms that can be introduced by means of a definition, there can be at most countably many of those. In the first case, because they would be finite inscriptions built from letters

\begin{abstract}
11 I have not encountered an argument formulated exactly this way, but it seems that the tendency can be traced back at least to Quine. In (1947) he remarks that any usage of general terms in the context of quantification commits one to abstract objects (pp. 74-75), and he explicitly says about quantifiers binding predicate letters:

If we bind the schematic predicate-letters of quantification theory, we achieve a reification of universals which no device analogous to Fitch's is adequate to explaining away. These universals are entities whereof predicates may thenceforward be regarded as names; they may be construed as attributes or as classes ... The predicate letters, when thus admitted to quantifiers, acquire the status of variables taking classes as values (pp. 77-78).
\end{abstract}

He is even more explicit when he discusses Leśniewski's Ontology (Quine 1952, p, 141). There, he criticizes Ajdukiewicz and Leśniewski for not attaching sufficient significance to the fact that "the variables which have been said to stand in places appropriate to general terms are subjected in Leśniewski's theory to quantification”. This theory, according to Quine, “...surely commits Leśniewski to a realm of values of his variables of quantification; and all his would-be general terms must be viewed as naming these values singly". Quite rhetorically Quine claims that if this quantification is not to be read as committing Leśniewski to classes, then he is "at a loss to imagine wherein such commitment even on the part of a professing Platonist can consist”. Quine's argument is, however, slightly different from the one I gave. He is already assuming that being subjected to quantification automatically carries ontological commitment, and instead of explicitly discussing the need for semantics and how what semantics we choose can impact our ontological commitment he insists that sentences should be put in their standard first-order form. Quine (1970, pp. 66-67) contains yet another argument. In ordinary first-order quantifications (like the one expressed by ' $(\exists x)(x$ walks $)$ ') the open sentence displays a variable in a position where a name could stand. The quantified sentences are not read as quantifying over names: they rather quantify over objects that could be named by a name placed in the position of a variable. Similarly when we bind predicate variables, we "treat predicate positions suddenly as name positions, and hence ...treat predicates as names of entities of some sort". This argument has been dealt with by Boolos (1998b, pp. 37-39), who points out that to put a predicate variable in a quantifier may require that the variable be treated as having a range but we do not have to treat predicate positions as name positions just because we do so for ordinary individual variables.

A train of thought which more explicitly relies on the relevance of the choice of a semantics can be found in (Burgess 2004, p. 217):

...if a model theory has not yet been developed of a given logical notion, it may be alleged that the notion is 'meaningless' because it lacks a 'semantics'. On the other hand, once a model theory has been developed for a given logical notion it may be alleged that problematic 'ontological commitments' are implicit in the use of the notion...

12 Actually, the authors argue that Ontology cannot be given substitutional semantics, and since they focus on the part of Leśniewski's Ontology which resembles QNL (i.e. they discuss quantification over name variables only), their arguments, if compelling, Mutatis mutandi apply here. 
from a finite alphabet (this is how a nominalist should think of names, arguably). In the second case, because definitions are finite inscriptions over a finite alphabet. This leads to a problem when we want to emulate set-theoretic second-order quantification over an infinite domain, for there are uncountably many subsets of each such domain, and thus we will run out of names supposed to go proxy for subsets. This indicates that it will be hard to run certain mathematical theories. For instance, it will be very hard to mimic the quantification over real numbers using this sort of substitutional reading. Also, even if the domain of objects is denumerable, the substitutional interpretation will give us a different result than the set-theoretic one, because there will be more subsets of the domain than possible names.

Let us call the arguments against any substitutional interpretation of higher-order (or plural) quantification which in one way or another relies on the claim that since the class of substituents is at most countable (and this constitutes a problem because we do not have any upper limit on the size of the domain of objects to be named) size limitation objections.

[SA4] relies on the size limitation objection and assumes that the only nominalistically acceptable approach to substitutional semantics for QNL is the one that puts the countability requirement on the set of names that can be substituted. In what follows, however, I will argue that there is a way of circumventing the intuitions underlying [SA4]. I will develop a modal-substitutional semantics for plural quantification to argue that the disjunction in [SA3] isn't as exhaustive as it may initially seem.

\section{Relational semantics for plural quantification}

In the relational semantics for QNL the quantifiers range over certain ways names could be. The relational models defined below will introduce the notion formally in a fairly well-known framework. This will allows us to get a better handle on the semantics and hopefully provide us with a better understanding of this notion.

Suppose we start with a world which for our purposes is devoid of linguistic objects (especially names). The content of this world is our domain of (already existing) extralinguistic individuals, call it the bare world (mind you, it doesn't have to be the domain of all extra-linguistic objects; it just involves an arbitrarily selected domain of base objects that we decided to be concerned with). In such a world, it is possible to introduce names which would either be empty or refer to one or more already existing objects. In this setting we can take a possible world to consist of two sorts of objects: bare individuals (those which do not name anything) and names that either do not name anything or name (one or more) extralinguistic individuals. The situation in a possible world determines what reference the names that exist in it have (if there are any). The bare world can be also interpreted as such a possible world, only at least one of the sorts would be empty. Starting with the bare world we can subsequently extend its repertoire of names by introducing (countably many) new names that refer to bare individuals. Let us idealize here: we are not putting any restrictions on which individuals can be named by a name, we assume that any bare individual (or any bare individuals) is (are) nameable. This gives raise to the so-called naming structure: 
Definition 1 A naming structure is a tuple $\langle I, W\rangle$ where $I$ is a set (of bare individuals) and $W$ is a set of possible worlds. A possible world is a tuple $\langle N, \delta\rangle$ where $I \cap N=\emptyset$ and $\delta \subseteq N \times I$. A bare world is the possible world with $N=\emptyset$. The following conditions all have to be satisfied:

(i) $B=\langle\emptyset, \emptyset\rangle \in W$ (i.e. the naming structure contains a bare world).

(ii) For any $w \neq B, w=\langle N, \delta\rangle, N$ is countable and $N \neq \emptyset$.

The accessibility relation on possible worlds is defined by the following condition. Let $w=\langle N, \delta\rangle, w^{\prime}=\left\langle N^{\prime}, \delta^{\prime}\right\rangle . R w w^{\prime}$ if and only if $N \subset N^{\prime}$ and $\{\langle x, y\rangle \mid x \in$ $\left.N \wedge\langle x, y\rangle \in \delta^{\prime}\right\}=\delta$ (i.e. $\delta^{\prime} \cap N \times I=\delta$ ).

If $\mathbf{M}$ is a naming structure and $I$ is the set of its bare individuals, I say that $I$ underlies $\mathbf{M}$ or that $\mathbf{M}$ is based on $I$. If $\mathbf{M}=\langle I, W\rangle$ and $w \in W$ I will sometimes write $w \in \mathbf{M}$.

A few words of explanation here. First, we start "constructing" a naming structure with a bare world. This bare world together with $I$ represents the situation where we have a set of objects that can be named but we have not introduced any names yet. Since in principle (unless one has very specific religious beliefs) there is no reason to believe in unnameable individuals, the $I$ in the bare worlds is simply the domain of objects.

We are considering ways individuals which are not names could be named. This means that introducing new names does not change the domain of individuals (that is why $I$ is a set in the naming structure and does not vary with possible worlds). Also, this indicates that the only way we get from one possible world to another accessible world is by extending the repertoire of available names (so $N$ has to be a proper subset of $N^{\prime}$ ).

On the nominalist reading, quite plausibly, names are finite inscriptions composed of symbols (or phonemes) from a finite alphabet. This means that in any possible world there can be at most countably many names (hence the requirement that $N$ be countable). Moreover, the basic idea is that by "going" to another possible world we are extending what we already have; not changing the ways that names that we already have are. That is, we can add a new name and take it to refer to such-and-such objects, but the reference of the already existing names cannot be changed. In a sense, names are thought of as given with reference. Hence the denotation relation in an accessible world has to agree with the initial denotation relation on all names that already existed before we extended the set of names.

This sort of structure will not ensure yet that every intuitively possible "way of meaning" will have a representation in a model. To do this, we have to require that for any subset of the domain of individuals in a possible world there be an accessible possible world in which there is a name which denotes all and only its elements.

Definition 2 Let $\langle N, \delta\rangle=w \in W$. A naming structure $\mathbf{M}=\langle I, W\rangle$ is $w$-complete if and only if:

$$
\forall A \subseteq I \exists w^{\prime}=\left\langle N^{\prime}, \delta^{\prime}\right\rangle\left(R w w^{\prime} \wedge \exists x \in N^{\prime} \forall y \in I\left(\delta^{\prime}(x, y) \equiv y \in A\right)\right),
$$

$\mathbf{M}=\langle I, W\rangle$ is complete iff for any $w \in W \mathbf{M}$ is $w$-complete. 
The basic idea is that a naming structure is $w$-complete iff for any set of individuals existing in this world, there is a world accessible from $w$ where a name which names elements of this set exists. In other words, it is $w$-complete if it models the full range of ways names of objects from $w$ could be.

The reason why $B$-completeness is not sufficient is that the class of $B$-complete naming structures would agree with classical set-theoretic semantics only on sentences with only one quantifier. In a naming structure, the iteration of quantifiers carries us deeper and deeper into the structure. ${ }^{13}$

I can now explain how quantification in QNL is supposed to range over ways names could be (or refer). I will be evaluating sentences in a name structure by evaluating them in its bare world. First, we have to define satisfaction of a formula at a world in a naming structure.

Definition 3 An $\mathbf{M}$-interpretation is a triple $\langle\mathbf{M}, w, v\rangle$, where $\mathbf{M}$ is a naming structure, $w=\langle N, \delta\rangle$ is a possible world in $\mathbf{M}$ and $v$ either assigns to every variable in QNL an element of $N$, if $N \neq \emptyset$, or is the empty function on the set of variables of QNL otherwise. If $\mathbf{M}$ is a complete naming structure, then we say that this $\mathbf{M}$-interpretation is complete.

Thus, an interpretation picks a naming structure, a possible world in it, and if there are any names in this world, it assings names to variables. Satisfaction of formulas under M-interpretations is defined as follows.

Definition 4 Let $\langle\mathbf{M}, w, v\rangle$ be an M-interpretation, $w=\langle N, \delta\rangle$. Also, let $a$ and $b$ be QNL-variables and $\phi$ and $\psi$ be QNL-formulas. The relevant satisfaction clauses are:

$\langle\mathbf{M}, w, v\rangle \models_{\diamond} a \underline{\mathrm{b}}$ iff $v(a)$ and $v(b)$ are defined, $\exists !_{x \in I}\langle v(a), x\rangle \in \delta$, and $\exists y \in I(\langle v(a), y\rangle \in \delta \wedge\langle v(b), y\rangle \in \delta)$.

$\langle\mathbf{M}, w, v\rangle \models_{\diamond} \neg \phi$ iff $v$ is not the empty function and $\langle\mathbf{M}, w, v\rangle \not \models$.

$\langle\mathbf{M}, w, v\rangle \models_{\diamond} \exists a \phi$ iff for some $w^{\prime} \in M, R w w^{\prime}$ and $\left\langle\mathbf{M}, w^{\prime}, v^{\prime}\right\rangle \models \phi$

where $v^{\prime}$ differs from $v$ at most in what it assigns to $a$.

(I skip the clause for conjunction because it is fairly standard, but I give the clause for negation because it is slightly different from the usual formulation.)

The basic idea is that we treat the class of names that exist in a certain world as the class of substituents when we evaluate a formula in this world. Since the bare world does not contain names the notion of satisfaction in the bare world as applied to open formulas is not very fascinating. No formula containing free variables will be satisfied at the bare world.

In a non-bare world $w$ an atomic formula $a \varepsilon b$ is satisfied if the first one is assigned a singular term referring to an object which is among the objects referred to by the name assigned to $b$ (this includes the "degenerate" case where the name assigned to $b$ also refers to exactly one object). A negation of a formula is satisfied iff the formula

13 The notion of B-completeness is just the notion of $w$-completeness applied to a particular world $B$. 
isn't satisfied (the non-emptiness clause is there only to handle the bare world, so it is irrelevant now). An existentially quantified formula is satisfied iff $w$ can access $w^{\prime}$ (that is, the way names are in $w$ can be extended to the way names are in $w^{\prime}$ ) and a name being a witness to that quantifier exists in $w^{\prime}$.

When it comes to evaluating sentences a $a$ sentence is true in a naming structure $\mathbf{M}$ if and only if it is satisfied in its bare world under any valuation (and while no formula is satisfied at the bare world, sentences are assessed in an intuitively correct way). A sentence is $\mathbf{M}$-valid if and only if it is true in any naming structure. A sentence is $M$-complete valid if it is true in any complete naming structure.

To see how this semantics works it is worthwhile to take a look at a few examples. I'll first give a claim, then its formal representation, and then its semantic interpretation expressed in English.

1. 'Socrates is a philosopher' is represented by 'Socrates $\varepsilon$ philosophers' and has the unsurprising truth condition that the name 'Socrates' has to refer to exactly one object being among the objects to which the name 'philosophers' refers.

2. 'All cats are animals' is represented by ' $\forall a(a \varepsilon$ cats $\rightarrow a \varepsilon$ animals)' and is true iff any possible name $a$ is such that if it refers to exactly one objects which is among the objects to which 'cats' refers, it is also among the objects to which 'animals' refers.

3. 'Some logicians admire only each other' is represented by

$$
\begin{array}{r}
\exists a[\forall b(b \varepsilon a \rightarrow b \varepsilon \text { logicians }) \wedge \\
\wedge \forall c \forall d(c \varepsilon a \wedge c \text { admires } d \wedge \\
\wedge d \varepsilon d \rightarrow \neg d \varepsilon c \wedge d \varepsilon a)]
\end{array}
$$

and is true iff there is a possible name $a$, such that:

(a) Every object to which $a$ refers is a logician (because any possible name $b$ which names one of the $a$ s also names a logician),

(b) any possible singular term $c$ which names one of the $a$ s, and any possible singular term $d$ which names an object admired by $c, d$ names an object already being one of the $a$ s.

Now, it is interesting to observe that as far as our assessment of sentences goes, this modal semantics gives the same results as standard set-theoretic semantics.

Theorem 1 The following correspondence holds:

(a) For any set-theoretic QNL model there is a complete $\mathbf{M}$-model which agrees with it on all formulas.

(b) For any complete M-model there is a set theoretic QNL model which agrees with it on all formulas.

Argument By model construction:

$A d(a)$. Suppose a formula $\phi$ fails at an S-model $M=\langle D, I n\rangle$, let $a_{1}, \ldots a_{k}$ be all variables that occur in $\phi$. Take a naming structure $w$ with $I=D$. Since the structure is complete, there exists a possible world $w=\langle N, \delta\rangle$ such that for 
any $A \subseteq D$, if for some $i, \operatorname{In}\left(a_{i}\right)=A$ then there is a $y \in N$ such that for all $x \in I, \delta(y, x) \equiv x \in A$. If $\operatorname{In}\left(a_{i}\right)=A$ let $v\left(a_{i}\right)$ be an arbitrary $y \in N$ for which this condition $(\forall x \in I(\delta(y, x) \equiv x \in A)$ holds. Then also $\phi$ fails at this possible world under $v$. The proof (that $M$ and $w$ agree on $\phi$ ) goes by induction. If, on the other hand, $\phi$ does not fail at $M$, then it also does not fail at any naming structure $w$ with $I=D$. If it did, then using the procedure described in (b) below we could show that it would also fail at the original S-model.

$A d(b)$. I will just show, given a non-bare world in a naming structure and a valuation function, how to construct a corresponding S-model that satisfies exactly those formulas that are satisfied at that world under that valuation. Take a $w=\langle N, \delta\rangle$ in a naming structure based on $I$. Let $v$ map name variables $a_{1}, a_{2}, \ldots$ into $N$. To obtain an equivalent $S$-model $w^{\prime}=\langle D, \operatorname{In}\rangle$ take $D=I$ and let $\operatorname{In}\left(a_{i}\right)=\{x \in I \mid$ $\left.\delta\left(v\left(a_{i}\right), x\right)\right\}$ for any $a_{i}$. The proof that two such models satisfy the same formulas is by induction.

The following observations are in order. First, modulo this relational semantics, QNL has an intuitive translation into a two-sorted first order quantified modal logic with one additional primitive binary operator representing the naming relation, which supports the idea that the commitment of QNL doesn't have to go beyond that of first-order modal logic (the translation is rather straightforward). Second, the size limitation objection does not apply here. Quantifiers are interpreted as ranging over possible names, but not over possible names from one particular possible world but rather over names that belong to the union of all sets of names from all accessible possible worlds. The initial plausibility of the objection results from the ambiguity between the following two readings:

[R1] It is possible that for every subset of the domain there is a name which names all and only those objects which are elements of this subset.

[R2] For any subset of the domain it is possible that there is a name which names all and only all its elements.

[R2] might hold even if [R1] fails. For instance, suppose the domain consists of all real numbers. It is false that there is a possible world in which all elements of the domain are named by individual terms, because there are not enough names in any particular possible world. However, this does not mean that there are unnameable real numbers. Quite the contrary, every real number can be named in some accessible possible world. The abundance of numbers arises from the abundance of accessible possible worlds - the ways names could be.

\section{Philosophical qualms}

Let's take a look at certain philosophical qualms about the nominalistic acceptability of this approach. The first objection focuses on the ontological commitment of the possible-world discourse. The second one emphasizes that the modal reconstructions fail to avoid the epistemological difficulties that realism runs into. The third one points out that while giving a nominalist reconstruction, I have employed set theory 
in metatheory. The fourth one questions whether the approach developed in this paper can handle the size limitation objection.

\subsection{Modality and the innocence of possible worlds}

An objection can be raised that while explaining away the quantification over sets I do it at the expense of taking on the commitment to possible worlds. This sort of objection has been raised by Woleński (1992) against Chihara (1990). ${ }^{14}$

Responding merely that possible worlds discourse is a convenient myth ${ }^{15}$ is not philosophically satisfactory: one needs to explain how such myths can be used in a philosophical account of the truth of serious theoretical statements. ${ }^{16}$

Ultimately, the nominalist, for the success of the current enterprize, should either provide a philosophically compelling nominalistic account of the possible-world talk, or to explain the modal notions involved without reference to possible worlds. While I do not dismiss the concerns, I think both goals can be achieved, but this paper is already long the way it is. For now, I will rest content with the conditional claim: if the nominalist can accomplish either of these two tasks, he can use higher-order quantification without commitment to abstract objects.

For a reader demanding an ultimate justification of the nominalist views this might seem unsatisfactory. Alas, the scope and focus of this paper force me to postpone a discussion of these issues to a different work. So instead of addressing the issue fully, I will just hand-wavily gesture towards the direction that I think considerations of this issue should go.

Given the multiplicity of anti-realist approaches to possible world discourse, it is far from obvious that the nominalist is committed to abstract objects just be cause he employs the possible-world talk. This is especially so, because the involved notion of possibility isn't as heavily theory-laden as various other modalities. The notion of nameability does not require the existence of persons who can actually name objects, nor does it require the real existence of other possible worlds where possible names exist. Certain objects are considered nameable if by introducing a name that multiply denotes exactly those objects we would not run into a paradox, and that is all there

\footnotetext{
14 There is some similarity between the modal account I propose and Chihara's use of constructibility quantifiers. There are some differences, however. When we extend the language to higher-order names (the strategy that lies beyond the scope of the present work, cf. Urbaniak (2008)), the result will not resemble type theory, but rather a cumulative hierarchy. Instead of introducing constructibility quantifiers explicitly I model ways names could be using standard modalities and reshaping the accessibility relation, which allows for more flexibility and captures the philosophical ideas in a more familiar framework. I also think that the framework I presented is easier to generalize to obtain a better nominalistic account of the language of set theory or arithmetic (especially the fact that mathematical terms behave like singular terms), but let's not concern ourselves with these issues here (see however Urbaniak (2010) for details).

15 "Remember, that for me, this whole possible world structure is an elaborate myth, useful for clarifying and explaining modal notions, but a myth just the same. It would be a mistake to take this myth too seriously and imagine that we are exploring real worlds, finding there real open — sentence tokens that have puzzling features". (Chihara 1990, p. 60)

16 Chihara has much more to say about possible worlds and modalities (see Chihara 1998), and he makes quite a strong case for the nominalistic acceptability of possible worlds talk.
} 
is to it. A theory of nameability thus understood is not a chapter in mathematical theology (if I may borrow an expression from Boolos et al. 2002, p. 19) concerned with ideal naming agents, other real worlds and so on, but rather a theory which tries to describe certain conditions under which a name that denotes certain objects can be introduced without worrying about consistency and paradoxes. Vivid metaphors which employ possible worlds and an ideal naming agent may be helpful in clarifying logical properties of the modalities involved, but otherworldly objects or the possible behavior of ideal agents are not what the theory is about, pretty much like the computability theory is not really about possible worlds where certain computations are performed.

Ultimately, perhaps, it would be better to avoid the possible-world talk in a nominalistic account of higher-order quantification. Instead, a nominalist could argue that the modality involved should be taken as a primitive and that the interpretation of quantifiers in QNL sentences boils down to phrases like 'it is possible to introduce a name (it wouldn't lead to a paradox if we introduced a name) which refers in suchand-such a way such that...'. But this, prima facie, does not commit one to abstract objects. In such a case, an axiomatization of the modal framework should be provided together with a sensible defense of its nominalistic acceptability.

\subsection{Epistemology and modality}

Shapiro (1993) suggests that the epistemological challenges to platonism (Benacerraf 1973) apply also to modal approaches:

At least prima facie, the epistemology of the various modal notions is more tractable than an epistemology of abstract objects like sets. The contention of this paper, however, is that this promise is not delivered. The epistemological problems facing the anti-realist programmes are just as serious and troublesome as those facing realism. Moreover, the problems are, in a sense, equivalent to those of realism. No gain is posted, and in some cases there is a loss (Shapiro 1993, p. 456).

The core of Shapiro's criticism seems to be this:

I show that there are straightforward, often trivial, translations from the settheoretic language of the realist to the proposed modal language, and vice-versa. The translations preserve warranted belief, at least, and probably truth (provided, of course, that both viewpoints are accepted, at least temporarily). Under certain conditions, the regimented languages are definitionally equivalent, in the sense that if one translates a sentence $\phi$ of one language into the other, and then translates the result back into the original language, the end result is equivalent (in the original system) to $\phi$. The contention is that, because of these translations, neither system can claim a major epistemological advantage over the other. Any insight that the modalist claims for his system can be immediately appropriated by the realist, and vice-versa. The problem, however, lies with the "negative" consequences of the translations. The epistemological problems with realism get "translated" as well (p. 457). 
Putting technical qualms about translations aside, the worry seems to be that the modal reconstruction, since it preserves theoremhood, is left with the truth of pretty much the same (modulo translation) set of truths to explain.

There is an important distinction to be made, if this argument is to be dealt with adequately (for simplicity let's focus on a particular theory, ZFC): the distinction between a mathematical theory whose truth is to be explained itself (ZFC itself), and the same mathematical theory together with the realist philosophical story about it (in our case, ZFC plus a Platonist account of sets, call it ZFC+).

The mathematical theory itself doesn't contain any philosophical claim about the ontological status of objects that it is about. Philosophical stories about the mathematical theory are sometimes given, but only by those who quite independently of the mathematical enterprise attempt to develop a deeper philosophical understanding of the mathematical apparatus.

Indeed, even most successful mathematicians using set-theory rarely have developed any deeper philosophical ontological considerations meant to interpret what they're doing. What's more, often different mathematicians when asked philosophical questions about set theory, either explicitly don't care or give completely different answers. This suggests that to use ZFC as a successful mathematical tool one doesn't need any deep philosophical understanding of the language of set theory, pretty much like to spill ink one doesn't have to have read and understood Austin's “Three ways of spilling ink" (Austin 1966).

Now suppose one gives a modal-nominalist account of ZFC: a translation of the language of ZFC into some (not necessarily really different) language, a certain theory (call it NZFC) such that ZFC and NZFC are the same under such translation ${ }^{17}$ and a nominalistic philosophical account of the truth of NZFC (let's dub NZFC with this explanation $\mathrm{NZFC}+$ ).

Is the fact that NZFC is modulo translation pretty much the same as ZFC a serious objection against the nominalistic acceptability of this philosophical explanation? I would like to submit, it isn't. If the mere agreement on theorems of ZFC and NZFC were to bring in the same epistemological worries as those that $\mathrm{ZFC}+$ encounters, any nominalistic attempt to reconstruct ZFC would be pointless to start with. This, however, seems hasty and far from obvious. Even if nominalistic reconstructions are doomed, it is unlikely that it is mere theoremhood preservation per se that brings doom.

If NZFC+ preserved the truth of $\mathrm{ZFC}+$, that would be a serious problem for the nominalist. The thing is, it isn't supposed to do that. Quite to the contrary, the nominalist doesn't want to preserve the truth of ZFC+ minus ZFC. He explicitly disagrees with it.

In general, the philosophical significance of interpretability of formal theories is a tricky issue and it is far from obvious that a mathematical interpretability result automatically shows that any philosophical account of the interpretans has to inherit the issues encountered by the Platonist's favorite philosophical account of the interpretandum. For instance, it is rather hasty to claim that there is a strong philosophical connection between real analysis and geometry just because the former can be interpreted in the latter.

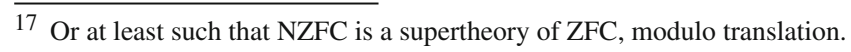


Perhaps, a more general worry still stands: why is it that the modal notions are regarded as more tractable than the Platonist account, and yet yield support to the same mathematical theory? Well, this depends on what we mean by tractability.

If tractability is measured in terms of strength of mathematical theories whose truth is being philosophically explained, then sure, any good reconstruction should have (more or less) the same level of tractability. But, for the reasons described above, it is far from obvious that tractability thus understood is a measure of epistemic viability of the associated philosophical account.

If tractability is rather taken to capture our intuitions about relative viability of the associated philosophical accounts, then mere theoremhood preservation of mathematical theories doesn't have to preserve tractability, and since no mathematically tractable notion of tractability thus understood has been developed, we have to rely on our philosophical intuitions and judge these things on case-by-case basis.

That being the case, I submit that the account of modalities given in the previous subsection is philosophically less demanding than Platonism. We might have good reasons to believe that introduction of a certain name satisfying such-and-such conditions wouldn't lead to contradiction, because we've played around with various paradoxical arguments enough to figure out that as long as our naming hierarchy is grounded, we're safe. On the other hand, no amount of time spent on doing logic or mathematics seems to move us towards having better epistemic access to causally inert abstract objects as pictured by the Platonist.

\subsection{Can we use set-theory in metatheory?}

A related worry shines through some of Shapiro's remarks:

To be sure, the modal notions invoked by our antirealists do have uses in everyday (non-mathematical) language, and competent speakers of the language do have some pre-theoretic grasp of how they work. But this grasp, by itself, does not support the extensively detailed articulation of the modal notions as they are employed by our anti-realists in their explications of mathematics. I think we do have a decent grasp of the extensive notions, but this understanding is not pre-theoretic. Rather, it is mediated by mathematics, set theory in particular. For example, one item concerns the relationship between model theory and the intuitive notion of logical consequence, in all its modal, semantic, and epistemic guises. Of course, everyone who reasons makes use of logical consequence, but some ontologists give the notion of consequence a central role in their philosophical stories. And most contemporary philosophers have come to accept the model-theoretic explication of consequence, which is executed in set theory, with all its ontology. The question at hand concerns the extent to which our anti-realists are entitled to the hard won model-theoretic results (Shapiro 1993, p. 457).

The problem seems to be that despite the fact that we do have some pre-theoretic understanding of the modalities at play, the nominalist still needs to use model theory to elucidate her understanding of the consequence operation involved, and this set theory is formulated in set-theory, which bears commitment to sets. 
One way to read the argument is to take it to proceed from [C1] to [C2]:

[C1] Set theory is used (indispensably) in the metatheory of a logic.

[C2] This logic is committed to the existence of sets.

But this is not a compelling strategy. After all, set theory is also used in the metatheory of first-order logic and no one claims that by the same token first-order quantification commits one to the existence of sets.

On a more charitable and weaker reading, the worry is that the modal semantics for QNL is motivated by nominalism, and it is suspicious that someone who cares about nominalistic acceptability of QNL doesn't mind using set theory in meta-theory. Suspicious as it may be, it is not incoherent. Someone might be a mathematical realist in general, and claim merely that it is not with QNL that the commitment to abstract objects kicks in. But sure, this is not a standard position either.

A more uniform response on the part of the nominalist might be that she indeed doesn't believe in abstract objects in general. Still, there is a sense in which set-theory is still available to her in a kind of reductio argument. If she intends to convince someone who doesn't mind using set theory about the ontological innocence of QNL, she is free to use set-theory, no matter what she thinks about its nominalistic acceptability.

This rather cheap strategy, although not completely insane, tends to backfire. If the nominalist really thinks set theory carries commitment to abstract objects, then even if the Platonist might become convinced about the innocence of QNL, the argument won't work for the nominalist herself and won't provide her with what she herself could take as a philosophically relevant account of QNL quantification.

Another reply on the part of the nominalist might be to say that the set theory employed in the description is very weak, and that in fact the intuitive explanation of the semantics might be equally well given in a metalanguage employing modally understood QNL-like quantification instead of set-theory talk. Arguably, using intuitively understood QNL quantification in the metalanguage while giving a semantics for QNL quantification in the object language is not worse than giving semantics for first-order quantifiers in a metalanguage in which first-order quantification already can be expressed.

Here is an example of how such a description could start:

- Read 'a possible world is a tuple composed of a set of individuals, a set of name tokens, and a reference relation between these two' as 'a possible world contains individuals and name tokens which refer to individuals'.

- Read the important part of Definition 1 as 'To evaluate formulas, we need to be told what individuals exist and what possible worlds can be considered. Among the latter, we need to include a possible world in which no names exist, and any other possible world has to contain countably many names. One possible world can access another if the latter contains all the names that the former does (preserving what they refer to), and at least one new name'.

- To ensure completeness of our evaluation framework, we also need to assume that for any possible world $w$, for any individuals, there is a possible world $w^{\prime}$ in which a name exists which refers to exactly these individuals.

Developing this alternative semantics in full, alas, lies beyond the scope of this already long paper. 
My problem with this strategy is that while it seems to work for QNL, it might be quite difficult when it comes to extending the nominalist account to richer mathematical frameworks.

Another strategy, which I find more compelling, is for the nominalist would be to rely on the distinction between a mathematical theory and the mathematical-theorycum-philosophical-account bundle, already employed in Sect. 7.2. On this approach, the fact that the nominalist uses ZFC (or NZFC for that matter) does not make her committed to abstract objects, as long as using NZFC and additional philosophical consideration, she is able to develop a compelling NZFC+.

Of course, this is nowhere close to getting off Neurath's ship. The point is, however, that it is philosophically respectable to use NZFC (but not ZFC+) and some philosophical ideas to explain how one can interpret NZFC (or simply ZFC) in a nominalistically acceptable way (just as it is respectable to use English and certain philosophical ideas to develop a philosophy of language that applies to English itself). There is no circularity involved here: it is ZFC with extra philosophical content that is supposed to deliver the nominalistic acceptability of ZFC.

A prima facie problem with this strategy is that it ultimately hinges on the availability of a plausible nominalistic interpretation of ZFC. Indeed, a nominalistic interpretation of ZFC is still to be given. I believe such an account can be given. ${ }^{18}$

Yet, it is unfair to say that the nominalist fails in providing a plausible nominalistic story about QNL quantification just because she uses ZFC in model theory. The nominalist is in the process of developing a philosophical account of ZFC using (among other things) ZFC itself. Explaining why plural quantification is non-committing is only a step towards this goal, and this explanation should be evaluated as such a step. ${ }^{19}$

To avoid misunderstandings, let me recap the slightly twisted dialectics of this section:

- The argument that by using QNL one is committed to sets because the metatheory of QNL uses sets fails. Semantics of first-order logic also employs some set theory, but it doesn't make any user of FOL committed to the existence of sets.

- One might still worry about the fact that a nominalist uses some set-theoretic language in his account of how his system works.

- To this one might respond that they're only nominalist about the commitment of QNL and quite happy about commitment to sets arising for some other reasons. This response I find unsatisfactory because ultimately my motivation for defending QNL is nominalism in general.

- Another strategy would be that a nominalist uses set theory in metatheory as a means of a reductio argument. I don't think this is ultimately viable because if

\footnotetext{
18 This issue lies beyond the content of this paper (see Urbaniak (2010) for a sketch of such a nominalistic strategy; or consider an account given by Hellman (1989).

19 One could ask: if the nominalist can use ZFC in her account of QNL quantification, why not use standard set-theoretic semantics to start with? The reason why this wouldn't work is that this wouldn't contribute to showing that QNL quantification is nominalistically acceptable. In contrast, on the current approach it is emphasized that the reading of QNL formulas does not involve reference to sets: 'it is possible to introduce a name token' is more clearly nominalistically acceptable than 'there exists a set'.
} 
that's the case, then the nominalist still owes us a literal account of what he think really is going on in his metatheory.

- Finally, my preferred strategy is to take the availability of ZFC as a mathematical theory as independent of deeper metaphysical stories one has to say about ZFC. On this approach, one can use ZFC together with some philosophical explanations to provide a deeper understanding of ZFC, just like one can use physics to philosophize about physics.

\subsection{What about the size limitation objection?}

We can recall that one of the objections against the substitutional interpretation of QNL was that if the domain is infinite we will always run out of substituents for under no circumstances the existence of uncountable infinity of names is possible. Naming structures by definition can only contain possible worlds with a countable number of names. It does not follow, however, that we will run out of possible names if the bare world is infinite. In any complete naming structure any subset of the domain will have a possible name corresponding to it. Of course, it is not the case that there is a possible world which contains all such names simultaneously, but that is not a problem for the modal interpretation of higher-order quantification. The initial plausibility of the objection results from the ambiguity between:

(7.3) It is possible that for every subset of the domain there is a name which names all and only those objects which are elements of this subset.

and

(7.4) For any subset of the domain it is possible that there is a name which names all and only all its elements.

Let us grant that the set of names in a possible world has to be countable; at least on the nominalist view names are finite inscriptions or utterances over a finite alphabet. The difference between the readings given above is pretty much like the difference between the statement that it is possible that every real number has a name (which would require the existence of uncountably many names) and the claim that for any real number it is possible for it to have a name. The first is false, the second true. Size limitation objections may work well against (7.3). But it is not (7.3) but rather (7.4) that is posited in the modal interpretation under discussion, and it is not prone to this sort of difficulties.

\section{Wrapping up}

There are other issues, which I was unable to discuss in this paper due to space restrictions. For now I will just rest content listing them:

- I think the proposed semantics is more suitable for a nominalist that a semantics developed by Boolos (1998a).

- One could argue that the completeness requirement suggests that the involved notion of possibility is quite strong. I think the objection is misled and relies on 
misreading logical possibility as some sort of "possibility of being named by a human being".

- Following Linnebo (2003), one could insist that elements such as full comprehension indicate that the theory has set-theoretic content. This, I think can be refused by pointing out that those elements, read modally, have nothing to do with sets and are quite innocent.

- One could argue that reference to multiple objects is not innocent, because names refer to multiple objects via sets. To my mind, this argument already assumes a strong claim about reference which requires an independent justification and which seems false to me anyway (because "dog" refers to particular dogs, not to an abstract object, the set of dogs).

- One of the objections to the strategy (Hochberg 1984) is that the account is not parsimonious because it now postulates more kinds of reference relation (singular and multiple). I fail to see how on my account there should be two types of reference relation instead of one, and I fail to see why (even if Hochberg was right) having two types of reference relation is nominalistically unacceptable.

After describing some standard reasons to think that second order quantification carries commitment to abstract objects, I developed a model-substitutional semantics of monadic second-order quantification to counter these allegation. The approach developed in this paper, as any other philosophical approach to anything, has its challenges, some of which I discussed in the previous section. The first objection pertained to the question of ontological innocence of the possible-world discourse. In response, I argued that the modal notions involved are ratherd light-weight compared to serious metaphysical modal notions. The second one assumed that the epistemological difficulties of realism get translated into the nominalist framework in one and the same package with whole mathematical theories. I argued that this criticism is too strong (it a priori excludes any successful non-realist account of a mathematical theory as failing to avoid the epistemological difficulties that realism runs into) and that merely mathematical notion of translation is to weak to preserve philosophical issues surrounding the relevant theories. The third one picked on the use of set theory in metatheory. I argued that this doesn't make the approach strictly speaking inconsistent, and sketched a few routes out for the nominalist. Then, I explained how the modal approach avoids the size-limitation objection.

Acknowledgments I pursued research contributing to this paper while being a British Academy Visiting Scholar at Bristol University and I thank British Academy for its support. I am also grateful to my host in Bristol, Øystein Linnebo, whose support has been invaluable. I would also like to express my gratitude to various audiences participating in discussions of earlier versions of this paper.

Open Access This article is distributed under the terms of the Creative Commons Attribution License which permits any use, distribution, and reproduction in any medium, provided the original author(s) and the source are credited.

\section{References}

Austin, J. L. (1966). Three ways of spilling ink. Philosophical Review, 75(4), 427-440.

Benacerraf, P. (1973). Mathematical truth. Journal of Philosophy, 70(19), 661-679. 
Boolos, G. (1998a). Nominalist platonism. In R. Jeffrey (Ed.), Logic, logic, and logic (pp. 73-87). Cambridge: Harvard University Press.

Boolos, G. (1998b). On second-order logic. In R. Jeffrey (Ed.), Logic, logic, and logic (pp. 37-53). Cambridge: Harvard University Press.

Boolos, G., Burgess, J. P., \& Jeffrey, R. C. (2002). Computability and logic (4th ed.). Cambridge: Cambridge University Press.

Burgess, J. P. (2004). E Pluribus Unum: Plural logic and set theory. Philosophia Mathematica, 12, $193-221$.

Chihara, C. S. (1973). Ontology and the vicious-circle Principle. Ithaca, NY: Cornell University Press.

Chihara, C. S. (1990). Constructibility and mathematical existence. Oxford: Oxford University Press.

Chihara, C. S. (1998). The worlds of possibility: Modal realism and the semantics of modal logic. Oxford: Oxford University Press.

Dunn, J. M., \& Belnap, N. D. (1968). The substitution interpretation of the quantifiers. Nous, 2(2), 177-185.

Hazen, A. (1993). Against pluralism. Australasian Journal of Philosophy, 71, 132-144.

Hellman, G. (1989). Mathematics without numbers: Towards a modal-structural interpretation. Oxford: Oxford University Press.

Hochberg, H. (1984). Nominalism, platonism and "Being True of". In Logic, ontology and language. Essays on truth and reality. Munich: Philosophia Verlag

Kearns, J. (1969). Two views of variables. Notre Dame Journal of Formal Logic, 10, 163-180.

Kielkopf, C. (1977). Quantifiers in ontology. Studia Logica, 36, 301-307.

Küng, G. (1974). Prologue-functors. Journal of Philosophical Logic, 3, 241-254.

Küng, G. (1977). The meaning of the quantifiers in the logic of Leśniewski. Studia Logica, 36, 309-322.

Küng, G., \& Canty, J. T. (1970). Substitutional quantification and Leśniewskian quantifiers. Theoria, 36, $165-182$

Lejewski, C. (1954). Logic and existence. The British Journal for the Philosophy of Science, 5, 104-119.

Leśniewski, S. (1927). O Podstawach Matematyki, Wstęp. Rozdział I: O pewnych kwestjach, dotyczących sensu tez ‘logistycznych'. Rozdział II: O ‘antynomji’ p. Russella, dotyczącej ‘klasy klas, nie bȩdących wł asnemi elementami'. Rozdział III: O różnych sposobach rozumienia wyrazów 'klasa' i 'zbiór'. Przegląd Filozoficzny, 30:164-206. [On the foundations of mathematics. Introduction. Ch. I. On some questions regarding the sense of the 'logistic' theses. Ch. II. On Russel's 'antinomy' concerning 'the class of classes which are not elements of themselves'. Ch. III. On various ways of understanding the expression 'class' and 'collection' (Leśniewski, 1991, 174-226)].

Leśniewski, S. (1930). Über die Grundlagen der Ontologie. Sprawozdania z posiedzeń Towarzystwa Naukowego Warszawskiego, Wydziat Nauk Matematyczno-Fizycznych, 23, 111-132. (On the foundations of Ontology, (Leśniewski, 1991, 606-628)).

Leśniewski, S. (1931a). O podstawach matematyki, Rozdział X: Aksjomatyka 'ogólnej teorji mnogości pochodzaca z r. 1921. Rozdziat XI: O zdaniach 'jednostkowych' typu 'Aعb'. Przeglad Filozoficzny, 34, 142-170. [On the foundations of mathematics. Ch. X. The axiomatization of the 'general theory of sets' from the year 1921. Ch. XI. On 'singular' propositions of the tyle 'Aعb', (Leśniewski, 1991, 350-382)].

Leśniewski, S. (1931b). Über Definitionen in der sogenannten Theorie der Deduction. Sprawozdania z posiedzeń Towarzystwa Naukowego Warszawskiego, Wydział Nauk Matematyczno-Fizycznych, 24, 289309. [On definitions in the so-called theory of deduction, (Leśniewski, 1991, 629-648)].

Leśniewski, S. (1991). Stanisław Leśniewski. Collected Works (2 vols.). Dordrecht: Kluwer Academic Publishers. (Edited and trans: Surma, S., Srzednicki, J., Barnett, D. I. continuous pagination).

Linnebo, Ø. (2003). Plural quantification exposed. Nous, 37, 71-92.

Parsons, C. (2008). Mathematical thought and its objects. Oxford: Oxford University Press.

Prior, A. (1965). Existence in Leśniewski and Russell. In J. Crossley (Ed.), Formal systems and recursive functions (pp. 149-155). Amsterdam: North-Holland.

Quine, W. V. (1947). On universals. The Journal of Symbolic Logic, 12, 74-84.

Quine, W. (1952). Review: On the notion of existence. Some remarks connected with the problem of idealism, by Kazimierz Ajdukiewicz. The Journal of Symbolic Logic, 17, 141-142.

Quine, W. (1970). Philosophy of logic. Englewwod Cliffs, New Jersey: Prentice-Hall.

Rickey, F. (1985). Interpretations of Leśniewski’s ontology. Dialectica, 39(3), 181-192.

Sagal, P. (1973). On how best to make sense of Leśniewski's ontology. Notre Dame Journal of Formal Logic, 14(2), 259-262.

Shapiro, S. (1993). Modality and ontology. Mind, 102, 455-481.

Simons, P. (1985). A semantics for ontology. Dialectica, 39(3), 193-215. 
Simons, P. (1995). Lesniewski and ontological commitment. In D. Miéville, D. Vernant (Eds.), Stanislaw Lesniewski Aujourd'hui, number 16 in Recherches Philosophie, Langages et Cognition (pp. 103-119). France: Université de Grenoble.

Smith, P. (2009). Charles Parsons: Mathematical thought and its object. http://www.phil.cam.ac.uk/ teachingstaff/Smith/logicmatters/Resources/Parsons1.pdf.

Urbaniak, R. (2008). Reducing Sets to Modalities. In Reduction and Elimination. Proceedings of the 31st Wittgenstein Symposium (pp. 359-361).

Urbaniak, R. (2010). Neologicist Nominalism. Studia Logica, 96, 151-175.

Woleński, J. (1992). Review: Constructibility and mathematical existence by Ch. Chihara. History and Philosophy of Logic, 13, 233-234. 